\title{
Re-covering blood vessels by astrocytic endfeet after laser ablation
}

\author{
Hideaki Kubotera ${ }^{1}$, Hiroko Ikeshima-Kataoka ${ }^{1}$, Anna L Allegra Mascaro ${ }^{2,3}$, Francesco Pavone ${ }^{2}$, \\ Takafumi Inoue ${ }^{1}$
}

${ }^{I}$ Department of Life Science and Medical Bioscience, Faculty of Science and Engineering, Waseda University, Tokyo, Japan, ${ }^{2}$ European Laboratory for Non-Linear Spectroscopy, University of Florence, Florence, Italy, ${ }^{3}$ Neuroscience Institute, National Research Council, Pisa, Italy

Astrocytes are glial cells, which are most abundant in the central nervous system, and they extend numerous fine processes. Astrocytic endfeet cover almost all the blood vessels and are considered to play important roles such as maintaining the blood-brain barrier (BBB) integrity and providing nutrition from blood to neurons and keeping homeostasis in the brain. However, the functional roles of astrocytic endfoot are still controversial, because previous studies using genetically modified animals or other methods could not remove only astrocytic endfeet. In this study, we performed laser ablation and in vivo imaging with a two-photon laser scanning microscope (2-PLSM) on astrocytic endfeet covering blood vessels using transgenic mice expressing EGFP in astrocytes driven by the glial fibrillary acidic protein (GFAP) promoter. Since our laser ablation method with the 2-PLSM targets only a limited lesion without causing any visible damage to surrounding tissue, it allowed us to manipulate astrocytic endfeet covering blood vessels. We could dislocate astrocytic endfeet which covered blood vessels. Furthermore, we observed that stripped blood vessels were recovered by the same or other astrocytic endfeet within a few days after ablation. The re-cover by other astrocytes was observed irrespective of life-or-death of the ablated astrocyte and laser ablation loci, e.g., endfoot processes or astrocytic endfeet on blood vessels. In addition, dislocation of blood vessel-covering astrocytic endfeet did not cause breakdown of the BBB. These results suggest that astrocytic endfeet do not maintain the BBB integrity by themselves. Although laser ablation has been already performed on neurons, microglia and blood vessels, this study reports laser ablation on astrocytes for the first time. 